\title{
ARTíCULO \\ Pasiphaea barnardi (Decapoda, Caridea, Pasiphaeidae) en los fiordos australes de Chile
}

\author{
Pasiphaea barnardi (Decapoda, Caridea, Pasiphaeidae) in the southern fjords of Chile
}

\section{Guillermo Guzmán ${ }^{*}$}

\begin{abstract}
${ }^{1}$ Museo del Mar, Facultad de Recursos Naturales Renovables, Universidad Arturo Prat, casilla 121, Iquique, Chile. gguzman@unap.cl

*Dirección actual: Museo de Zoología, Universidad de Sao Paulo, Avenida Nazaré 481, Ipiranga, C.E.P. 04263-000, Sao Paulo SP, Brasil

Abstract.- The glass shrimps belonging to genus Pasiphaea are scarcely registered in Chilean waters, with just 5 of the 66 species recognized today. Of these Pasiphaea acutifrons is reported erroneously to some localities in different oceans, this due basically to an ambiguous diagnosis. Recently, a paper was published that analyze the taxonomic status of this species, limiting the range of variability of its diagnostic characters and restricting its distribution. In Chile $P$. acutifrons is distributed from Iquique to Magellan Strait, but the informed diagnostic characters are not in agree with those of this specie. By this reason is proposed a study to analyze the material referred as $P$. acutifrons with the object verify the status of this species in Chile. To this end, the material of Pasiphaeidae of 3 important carcinological collections in this country were revised, as well as new material collected in the CIMAR XIV expedition. The results of this study reveals that the specimens of $P$. acutifrons in fact belong to other species. One of these is Pasiphaea barnardi which is reported here by first time for Chilean Fjord, between Golfo de Penas and Concepcion Channel.
\end{abstract}

Key words: Pasiphaeidae, Chile, taxonomic status, biogeography

Resumen.- Los camarones vidrio del género Pasiphaea están pobremente representados en las aguas de Chile con sólo 5 de las 66 especies reconocidas a nivel mundial; de ellas Pasiphaea acutifrons ha sido citada erróneamente para diferentes localidades en diferentes océanos, debido básicamente a lo ambiguo de su diagnosis. En una reciente publicación se analizó el estatus de esta especie limitando la variación de los caracteres diagnósticos y restringiendo su distribución geográfica a Sudamérica. En Chile P. acutifrons es citada desde Iquique al Estrecho de Magallanes, sin embargo los caracteres diagnósticos informados no coinciden con aquellos indicados para esta especie. Por tal motivo se planteó el objetivo de reestudiar el material de $P$. acutifrons citado para Chile con el fin de verificar el estatus de esos registros. Para ello se analizaron los ejemplares de Pasiphaeidae depositados en 3 importantes colecciones carcinológicas de este país, así como el material colectado durante el crucero CIMAR XIV. Los resultados revelaron que los reportes de $P$. acutifrons corresponderían a otras especies, una de ellas concierne a Pasiphaea barnardi, especie que se describe por primera vez para la zona de los fiordos chilenos entre el Golfo de Penas y el Canal Concepción.

Palabras clave: Pasiphaeidae, Chile, estatus taxonómico, biogeografía

\section{INTRODUCCIÓN}

La familia de camarones pelágicos Pasiphaeidae está representada en Chile por 8 especies, 5 de las cuales pertenecen al género Pasiphaea (Retamal 2000, Guzmán 2008). Hasta antes de la década del 80, se registraban en Chile tan sólo 2 especies de este grupo; i.e., Pasiphaea acutifrons Bate, 1888 y P. dofleini Schmitt, 1932 (Retamal 1973), con posterioridad se incluyen a: P. magna Faxon, 1893 y P. chacei Yaldwin, 1971 (Wehrtmann \& Carvacho 1997) y P. americana Faxon, 1893 (Guzmán \& Wicksten 1998, Guzmán 2008).
P. acutifrons fue descrita originalmente para la zona magallánica y luego ampliamente citada para diferentes localidades en el Pacífico sur y noreste, central y oeste, Atlántico sur este y oeste así como en aguas circumantárticas (Boschi et al. 1981, Méndez 1981, Takeda \& Hatanaka 1984, Takeda 1986, Iwasaki \& Nemoto 1987, Retamal 1993, Guzmán \& Wicksten 1998, Retamal 2000). Muchos de estos registros tienen errores de determinación taxonómica, según ha quedado demostrado en trabajos posteriores (Hayashi 2006); desafortunadamente estos 
registros erróneos han contribuido a ampliar los caracteres diagnósticos de esta especie. Hayashi (2006) revisó el material tipo de P. acutifrons, delimitando así los caracteres para esta especie. Basado en el trabajo de Hayashi (2006), se hace necesario reestudiar los ejemplares citados para distintas localidades que han sido asignados a $P$. acutifrons.

Una especie ampliamente distribuida en aguas circumantárticas corresponde a Pasiphaea barnardi Yaldwin, 1971, originalmente descrita para Nueva Zelanda y posteriormente registrada para el sur de Australia, cercanías de Sudáfrica y hasta frente a Islas Malvinas en el Atlántico suroeste (Kensley 1977, Burukovsky \& Romensky 1987, Kensley et al. 1987, Burukovsky 1993). Los caracteres diagnósticos, tales como número de espinas en el borde posterior del telson, armadura de los primeros y segundos pereiópodos y quillas en los somitos abdominales, de esta especie se sobreponen a lo indicado erróneamente para $P$. acutifrons.

Durante noviembre del año 2008, el Comité Oceanográfico Nacional (CONA), organizó el crucero número 14 del programa 'Cruceros de Investigación Marina en Áreas Remotas (CIMAR)', canales

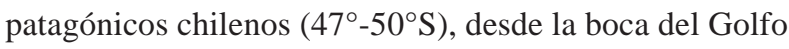
de Penas hasta el Canal Concepción por las aguas interiores de los fiordos. Se colectó material de esta familia de camarones, encontrándose al menos 3 especies (G. Guzmán, datos no publicados), una de las cuales corresponde a $P$. barnardi, nuevo registro para Chile y a través del presente trabajo se describe la especie, discutiendo las citas de $P$. acutifrons en Chile dada la redescripción realizada por Hayashi (2006).

\section{Materiales y Métodos}

El material de Pasiphaea barnardi analizado fue colectado durante el programa CIMAR XIV (organizado por el Comité Oceanográfico Nacional, CONA, de Valparaíso, Chile), realizado en los fiordos australes ente el Golfo de Penas (4659,40’S; $\left.75^{\circ} 41,77^{\prime} \mathrm{O}\right)$ y el Canal Concepción (5009,02’S; 7443,19’O), en el marco del proyecto ‘Estudio de la Fauna Mesopelágica del Mar interior entre Golfo de Penas y Canal Trinidad' (Meléndez et al. 2009). Los especímenes fueron capturados entre los 200 a los 1500 m de profundidad, usando una red de media agua tipo Isaac Kid Mid-water Trawl de $9 \mathrm{~m}^{2}$ de boca.

El material examinado fue preservado en alcohol 75\% a bordo de la embarcación y actualmente se encuentra depositado en la colección de referencia del Museo del
Mar de la Universidad Arturo Prat de Iquique, Chile bajo el acrónimo MUAP(CD).

Los especímenes fueron medidos con un vernier digital de 0,01 mm de precisión en su longitud del cefalotórax (lcf), desde el borde anterior (excluyendo la espina epigástrica) hasta la escotadura posterior del caparazón. En forma paralela, se analizó el material de camarones pelágicos, presente en las colecciones del Museo Nacional de Historia Natural de Santiago (MNHN), Chile y la Colección Carcinológica del 'Museo de Zoología de la Universidad de Concepción’ en Concepción, Chile (MZUC-CCC).

La identificación del material se basó en las descripciones originales de Yaldwin (1971) y la redescripción de Hayashi (2006), así como también en la comparación fotográfica con el holotipo de la especie.

\section{Resultados}

Pasiphaea baRnardi Yaldwin, 1971 (Fig. 1)

Sinonimia restringida (para mayores antecedentes ver Hayashi 2006 p. 206)

Pasiphaea barnardi Yaldwin, 1971

Phye pacificus - Stebbing, 1914

Pasiphaea meiringnaudei - Kensley, 1977

Pasiphaea acutifrons - Iwasaki \& Nemoto, 1987 (no Bate, 1888).

Pasiphaea berentsae - Kensley et al. 1987

\section{MATERIAL EXAMinado}

CIMAR XIV: 1 hembra (lcf = 29,44 mm), estación 5, MUAP(CD)-0458; 1 hembra (lcf = 11,44 mm), estación 7, MUAP(CD)-0459; 1 macho (lcf = 20,44 mm ) y 2 hembras (lcf = 27,47 y 29,68 mm) estación 17, MUAP(CD)-0460; 1 hembra (lcf = 11,94 mm), estación 19, MUAP(CD)-0461; 1 macho (lcf = 20,44 mm), estación 31, MUAP(CD)-0462; 1 macho $(\mathrm{lcf}=24,52 \mathrm{~mm}$ ) y 1 hembra $(\mathrm{lcf}=25,54)$ estación 35, MUAP(CD)-0463; 4 ejemplares, MZUC-CCC 16238; 2 ejemplares MZUC-CCC 31391; 1 ejemplar, MZUC-CCC 3598; 2 ejemplares, MZUC-CCC 16260; 1 ejemplar, MNHN 315; 1 ejemplar MNHN 437; 1 ejemplar, MNHN 668.

\section{Diagnosis}

Ejemplares de mediano tamaño; 11,44 a 29,68 mm de largo cefalotórax (lcf). Rostro o espina epigástrica alcanza 


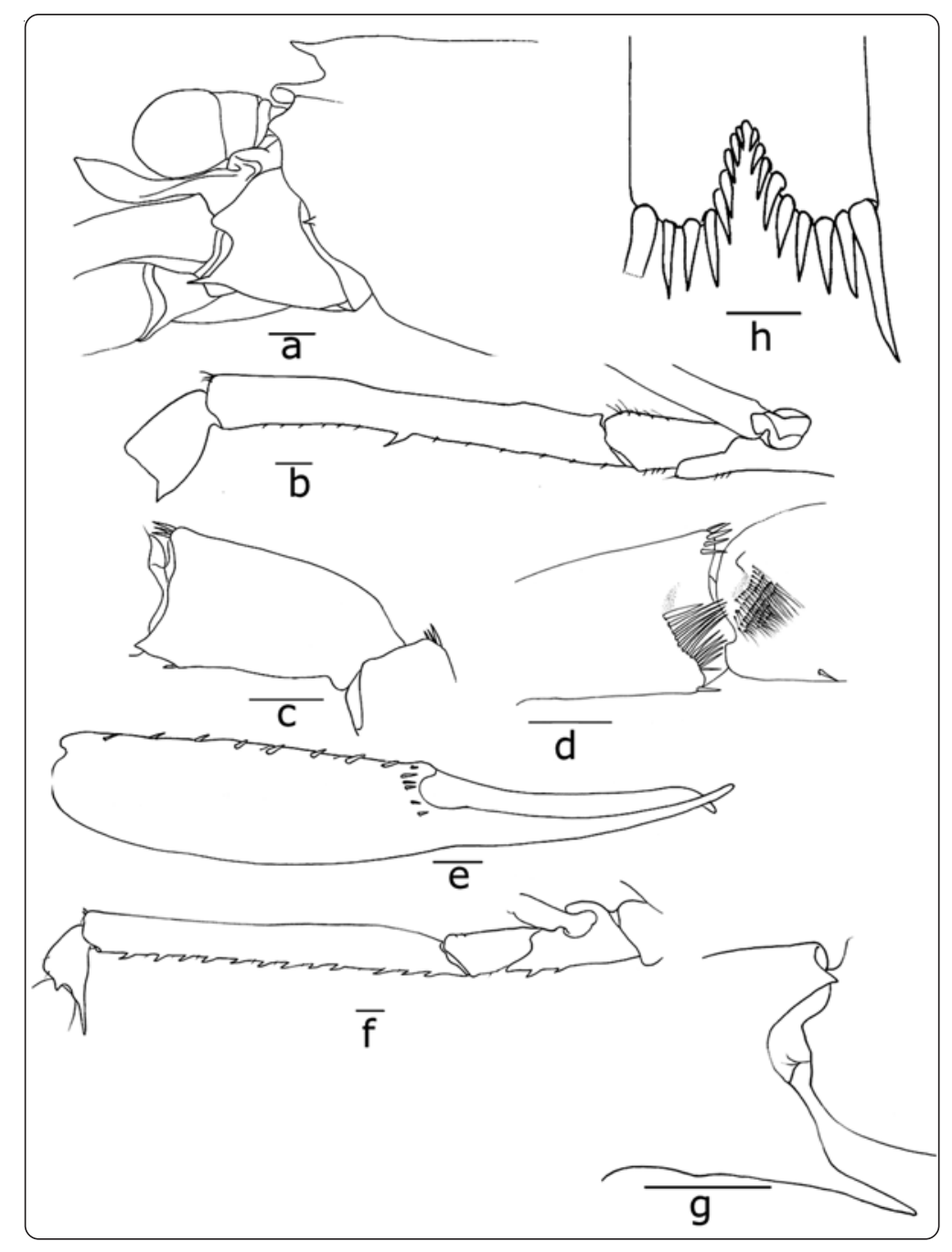

Figura 1. Pasiphaea barnardi. Ejemplar hembra 29,33 mm Icf. MUAP(CD)-0458. a) Región anterior del caparazón, b) Base, isquio, mero y carpo del primer quelípodo, c) Detalle de la cara externa del carpo del primer quelípodo, d) Detalle de la cara interna del carpo del primer quelípodo, e) Cara interna del primer quelípodo f) Base, isquio, mero y carpo del segundo quelípodo, g) Detalle del carpo del segundo quelípodo, $\mathrm{h}$ ) Porción posterior del telson. Barra de escala equivale a $1 \mathrm{~cm}$ (a, b, e y f) y $1 \mathbf{~ m m ~ ( c , ~ d , ~ g ~ y ~ h ) ~ / ~ P a s i p h a e a ~ b a r n a r d i . ~ F e m a l e ~ s p e c i m e n ~} 29.33 \mathrm{~mm}$ Icf. MUAP(CD)-0458. a) Anterior region of carapace, b) Basis, isquium, merus and carpus of first cheliped, c) Detail of the external face of carpus of first cheliped, d) Detail of the inner face of carpus of first cheliped, e) Inner face of first cheliped, f) Basis, isquium, merus and carpus of second cheliped, g) Detail of carpus of second cheliped, h: Posterior margin of telson. Scale bar: $1 \mathrm{~cm} \mathrm{(a,b,e} \mathrm{and} \mathrm{f)} \mathrm{and} 1 \mathrm{~mm}$ (c, d, g and h)

levemente el margen anterior del caparazón, borde anterior del mismo, cóncavo, con una espina aguda que apunta hacia arriba en un ángulo aproximado de $45^{\circ}$. Posteriormente se extiende en una carina dorsomedial que termina unos milímetros antes del borde posterior del caparazón. Espina branquiostegal presente, submarginal a marginal, que sobrepasa el borde anterior del caparazón. Seno branquiostegal poco profundo (Fig. 1a). Armadura del primer par de quelípodos, con un basipodito sin espinas, al igual que el isquiopodito (Fig. 1b). Meropodito con 3 a 4 espinas en el borde posterior (Fig. 1b). Carpopodito con una proyección distal redondeada, no como diente como en $P$. acutifrons, sino más bien como un lóbulo redondeado, con 5 cerdas espiniformes apicales (Fig. 1c). Región articular interna del carpopodito con el própodito con una depresión flanqueada por varias filas 
de espinas en la base de la quela y una fila de 11 cerdas espiniformes más una espina articulada en el margen distal del carpo (Fig. 1d). Palma de la primera quela con una fila de 9 cerdas en el margen dorsal interno, más 5 a 6 en la zona de unión con el dáctilo (Fig. 1e). Segundo par de pereiópodos con 2 a 3 espinas en el basipodito, más la espina o diente distal. Isquiopodito sin espinas ni proyección distal. Meropodito con 11 a 14 espinas en el borde posterior (Fig. 1f). Carpopodito con un fuerte diente ventral (Fig. 1g). Abdomen con pleonitos II al VI carinados en toda su longitud con una quilla notoria. Telson casi tan largo como el VI pleonito (95\% de su longitud). Borde posterior con una hendidura aproximadamente tan profunda como su ancho, armada con 9 pares de espinas (Fig. 1h).

Por otro lado, este reporte constituiría una ampliación de la distribución de Pasiphaea barnardi hacia el Pacífico austral Este. Aparentemente esta especie tendría una distribución circumantártica, al norte de la convergencia antártica, entre los $33^{\circ}-55^{\circ} \mathrm{S}$, a profundidades entre 0 y 1600 m (Fig. 2). Otras localidades reportadas: Estrecho de Cook (Yaldwin 1971), Nueva Zelandia, Nueva Gales del Sur, Australia (Kensley et al. 1987). Frente a Durban Sudáfrica (Kensley 1977). Burukovsky \& Romensky (1987) la citan para el Atlántico suroeste frente a las islas Malvinas (52 $05^{\prime}$ S; $57^{\circ} 02^{\prime} \mathrm{W}$ ) y en el Océano Indico al sureste de África (3306’S; 44³0’E) (Burukovsky 1993).

\section{Discusión}

Los ejemplares de Pasiphaea barnardi analizados coinciden con la diagnosis entregada por Hayashi (2006), así como también con la descripción original (Yaldwin 1971), difiriendo con esta última sólo en el número de espinas del meropodito del primer quelípodo (1 a 3 en el holotipo y hasta 4 en algunos ejemplares del CIMAR XIV y 2 a 3 en los ejemplares depositados en la colección del Museo de Zoología de la Universidad de Concepción). No obstante, Hayashi (2006) indica que la armadura del

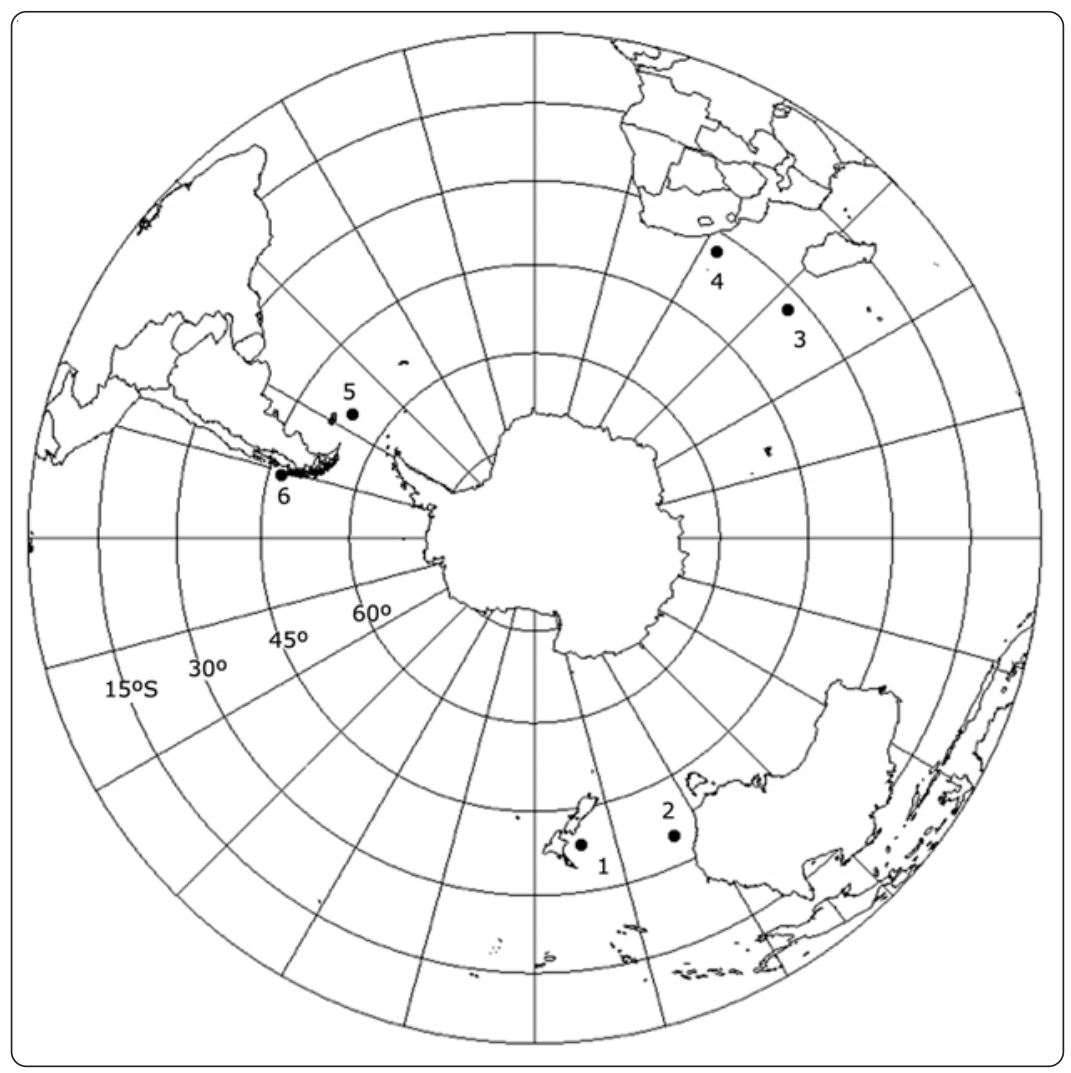

Figura 2. Mapa de la distribución de Pasiphaea barnardi Yaldwin, 1971. Los puntos y números indican los registros de esta especie. 1) Yaldwin 1971; 2) Kensley et al. 1987; 3) Burukovsky \& Romensky 1987; 4) Kensley 1977; 5) Burukovsky 1993; 6) este reporte. Los puntos son sólo referenciales de la localidad aproximada / Distribution maps of Pasiphaea barnardi Yaldwin, 1971. Dots and number show the published records of this species. 1) Yaldwin 1971; 2) Kensley et al. 1987; 3) Burukovsky \& Romensky 1987; 4) Kensley 1977; 5) Burukovsky 1993; 6) this report. Dots are only referential of the approximate locality 
meropodito en los paratipos de esta especie, tienen de entre 0 a 6 espinas, siendo lo más frecuente entre 0 a 4 . Los caracteres restantes se ajustan al rango dado para la especie (Yaldwin 1971, Hayashi 2006).

Morfológicamente $P$. barnardi es muy similar a $P$. acutifrons en la forma de la hendidura del telson, región anterior del caparazón e inclusive en la armadura de los meros de los quelípodos. Sin embargo, aparentemente la armadura del segundo par de quelípodos de $P$. acutifrons tendría de 5 a 9 espinas y no 11 como reporta la literatura (G Guzmán, datos no publicados).

Holthuis (1952) analizó los restos de una exuvia colectada durante la expedición LUND a Chile, capturada aproximadamente $10^{\circ}$ de latitud más al norte de la localidad tipo de $P$. acutifrons, indicando que corresponderían a $P$. acutifrons. No obstante, el espécimen posee 11 espinas en el margen posterior del mero del segundo quelípodo, carácter que coincide con lo observado en P. barnardi, por lo que es probable que el ejemplar de la expedición LUND, corresponda a esta especie.

En las muestras de media agua colectadas durante el CIMAR XIV no se registraron ejemplares de $P$. acutifrons, lo que plantea la interrogante de cuál sería el límite norte de la distribución para esta especie y, además, cuál es el rango de distribución geográfica, dado que es probable que los registros de $P$. acutifrons para el extremo sur de Sudamérica, hayan sido confundidos también con $P$. barnardi.

\section{Agradecimientos}

Se agradece al Programa CIMAR del Comité Oceanográfico Nacional y a toda la tripulación del AGOR Vidal Gormaz de la Armada de Chile. Al Dr. Pedro Báez del Museo Nacional de Historia Natural de Santiago, al Dr. Jorge Artigas y al personal del Museo de Zoología de la Universidad de Concepción. Al Dr. Marcos Tavares, y al Museo de Zoología de la Universidad de São Paulo, por el parcial financiamiento de este trabajo. A Nicole Olguín por sus comentarios a la redacción de este trabajo. A los amigos Margarita Ruiz-de-Gamboa y Marcos Ferrú. Este estudio forma parte del trabajo de tesis de doctorado del programa de postgraduación del Museo de Zoología de la Universidad de São Paulo y cuenta con el aporte de la Coordinação de Aperfeiçoamento de Pessoal de Nivel Superior (CAPES).

\section{LITERATURA CITADA}

Bate CS. 1888. Report on the Crustacea Macrura collected by H.M.S. Challenger during the years 1873-1876. Report on the Scientific Results of the Voyage of H.M.S. Challenger during the years 1873-1876. Zoology 24(2): 1-942, pls 150.

Boschi EE, MI Iorio \& K Fischbach. 1981. Distribución y abundancia de los crustáceos decápodos capturados en las campañas de los B/I “Walther Herwig” y "Shinkai Maru” en el mar Argentino, 1978-1979. Contribución, Instituto Nacional de Investigación y Desarrollo Pesquero 383: 233253.

Burukovsky RN. 1993. Shrimps of genus Pasiphaea (Crustacea, Decapoda, Pasiphaeidae) from the western part of the Indian Ocean. Byulleten Moskovskogo Obschchestva Ispytatelei Prirody, Otdel Biologicheskii 98(2): 33-40.

Burukovsky RN \& LL Romensky. 1987. Description of Pasiphaea balssi sp. n., a new species of shrimps from South Atlantic (Crustacea, Decapoda, Pasiphaeidae), and polytomous key for identification of the shrimps in the genus. Byulleten Moskovskogo Obschchestva Ispytatelei Prirody, Otdel Biologicheskii 92(6): 51-60.

Faxon W. 1893. Reports on the dredging operations off the west coast of Central America to the Galapagos, to the west coast of Mexico, and in the Gulf of California, in charge of Alexander Agassiz, carried on by the U.S. Fish Commission steamer 'Albatross', during 1891, Lieut.Commander Z.L. Tanner, U.S.N., commanding. VI. Preliminary descriptions of new species of Crustacea. Bulletin of the Museum of Comparative Zoology at Harvard College 24(7): 149-220.

Guzmán GG. 2008. Camarones pelágicos (Crustacea: Decapoda) en aguas del Pacífico sureste. Contribuciones al Estudio de los Crustáceos del Pacífico Este 5(1): 27-45.

Guzmán GG \& MK Wicksten. 1998. Nuevos registros de camarones de la familia Pasiphaeidae (Crustacea, Decapoda) en el norte de Chile ( $18^{\circ}$ a $22^{\circ}$ Lat. Sur, $70^{\circ}$ a $72^{\circ}$ Long W). Gayana Zoología 62: 203-210.

Hayashi KI. 2006. Revision of the Pasiphaea alcocki (WoodMason, 1891) species group with descriptions of two new species (Crustacea: Decapoda: Pasiphaeidae). In: Richer de Forges B \& J-L Justine (eds). Tropical deep-sea benthos 24. Memoires du Museum National d'Histoire Naturelle 193: 193-241.

Holthuis LB. 1952. Reports of the Lund University Chile Expedition 1948-1949. 5. The Crustacea Decapoda Macrura of Chile. Lunds Universitets Arsskrift. N. F. Afdelning, Série 2, 47(10): 1-109.

Iwasaki N \& T Nemoto. 1987. Distribution of pelagic shrimps in the Bering Sea and the northern North Pacific. Reports of the USA Marine Biological Institute, Kochi University 9: 233-239. 
Kensley B. 1977. The South African Museum's Meiring Naude cruises. Part 5: Crustacea, Decapoda, Reptantia and Natantia. Annals of the South African Museum 74: 13-44.

Kensley B, HA Tranter \& DJG Griffin. 1987. Deepwater decapod Crustacea from eastern Australia (Penaeidae and Caridea). Record of the Australian Museum 39: 263-331.

Meléndez RC, W Sielfeld \& G Guzmán. 2009. Informe de avance Proyecto Crucero CIMAR 14 Fiordos 'Estudio de la fauna mesopelágica del mar interior entre Golfo de Penas y Canal Trinidad'. Crucero CIMAR 14 Fiordos. Informes Preliminares. Comité Oceanográfico Nacional, Valparaíso, pp. 155-167.

Méndez M. 1981. Claves de identificación y distribución de los langostinos y camarones (Crustacea; Decapoda) del mar y ríos de la costa del Perú. Boletín Instituto del Mar del Perú 5: 1-170.

Retamal MA. 1973. Contribución al conocimiento de los crustáceos decápodos de la región magallánica. Gayana Zoología 29: 1-24.

Retamal MA. 1993. Crustáceos decápodos abisales de la zona Arica-Iquique. Estudios Oceanológicos 12: 1-8.
Retamal MA. 2000. Decápodos de Chile. ETI-Universidad de Concepción. Springer-Verlag, Berlín. [CD-ROM]

Schmitt WL. 1932. A new species of Pasiphaea from the Straits of Magellan. Journal of the Washington Academy of Sciences 22: 333-335.

Stebbing TRR. 1914. South African Crustacea (Part VII. of S. A. Crustacea, for the Marine Investigations in South Africa). Annals of the South African Museum 15: 1-55, pls 1-12.

Takeda M. 1986. Crustacea Decapoda. In: Nakamura I (ed.). Important fishes trawled off Patagonia, pp. 318-343. Japan Marine Fishery Resource Research Center, Tokyo.

Takeda M \& H Hatanaka. 1984. Records of decapod crustaceans from the southwestern Atlantic collected by the Japanese Fisheries Research Trawlers. Bulletin of the National Science Museum, Series A (Zoology) 10: 7-24.

Wehrtmann IS \& A Carvacho. 1997. New records and distribution ranges of shrimps (Crustacea: Decapoda: Penaeoidea and Caridea) in Chilean waters. Proceedings of the Biological Society of Washington 110: 49-57.

Yaldwin JC. 1971. Preliminary descriptions of a new genus and twelve new species of natant decapod Crustacea from New Zealand. Records of Dominion Museum 7: 85-94.

Recibido el 23 de mayo de 2013 y aceptado el 13 de noviembre de 2013

Editor: Claudia Bustos D. 